\title{
Coexistence of the antiferromagnetic and superconducting order and its effect on spin dynamics in electron-doped high- $T_{c}$ cuprates
}

\author{
Cui-Ping Chen, ${ }^{1}$ Hong-Min Jiang, ${ }^{1}$ and Jian-Xin $\mathrm{Li}^{1}$ \\ ${ }^{1}$ National Laboratory of Solid State of Microstructure and Department of Physics, Nanjing University, Nanjing 210093, China
}

(Dated: December 4, 2018)

\begin{abstract}
In the framework of the slave-boson approach to the $t-t^{\prime}-t^{\prime \prime}-J$ model, it is found that for electron-doped high- $T_{c}$ cuprates, the staggered antiferromagnetic (AF) order coexists with superconducting (SC) order in a wide doping level ranged from underdoped to nearly optimal doping at the mean-field level. In the coexisting phase, it is revealed that the spin response is commensurate in a substantial frequency range below a crossover frequency $\omega_{c}$ for all dopings considered, and it switches to the incommensurate structure when the frequency is higher than $\omega_{c}$. This result is in agreement with the experimental measurements. Comparison of the spin response between the coexisting phase and the pure SC phase with a $d_{x^{2}-y^{2}}$-wave pairing plus a higher harmonics term $(\mathrm{DP}+\mathrm{HH})$ suggests that the inclusion of the two-band effect is important to consistently account for both the dispersion of the spin response and the non-monotonic gap behavior in the electron-doped cuprates.
\end{abstract}

PACS numbers: 74.20.Mn, 74.25.Ha, 75.40.Gb

\section{INTRODUCTION}

The pairing symmetry of the hole-doped high- $T_{c}$ superconductors is generally believed to have the dominant $d_{x^{2}-y^{2}}$-wave pairing. However, the pairing symmetry of the electron-doped high- $T_{c}$ superconductors is still under debate $1,2,3,4,5,6,7,8$ While no consensus has been reached yet, more and more recent experimental results have suggested that the order parameter of electron-doped cuprates is likely to have a dominant $d_{x^{2}-y^{2} \text {-wave pairing }}$ symmetry, $,+4,5,6,7,8$ and with an unusual non-monotonic gap function.

Although various explanations have been proposed to account for the non-monotonic behavior, they can generally be categorized into two scenar$\operatorname{ios} \frac{5,7,9,10,11,12,13,14,15,16,17,18}{18}$ One is to extend the superconducting (SC) gap out of the simple $d_{x^{2}-y^{2} \text {-wave }}$ via the inclusion of higher harmonics term $\underline{5,7,9,10,11,12,13}$ From theoretical perspective, the non-monotonic $d_{x^{2}-y^{2}-}$ wave gap appears under the assumption that the $d_{x^{2}-y^{2}}$ wave pairing is caused by the interaction with the continuum of overdamped antiferromagnetic (AF) spin fluctuations. In this scenario, the non-monotonic gap behavior is described by the combination effect of a $d_{x^{2}-y^{2}}$-wave paring plus a higher harmonics term $(\mathrm{DP}+\mathrm{HH})$. Therefore, it is an intrinsic property of the SC state regardless of the presence of the AF order, and a simple one-band model is used to reproduce the non-monotonic gap behavior. The other argues that the non-monotonic behavior is the outcome of the coexistence of the $\mathrm{AF}$ and the SC orders $14,15,16,17,18$ This scenario assumes that the

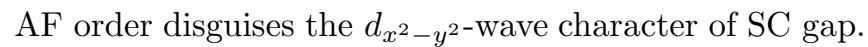
When the AF order is introduced, the resulting quasiparticle (QP) excitation can be gapped by both orders and behaves to be non-monotonic, although the SC gap itself is monotonic. The scenario gained support from the angle-resolved photoemission spectra (ARPES) mea- surements, where two inequivalent Fermi pockets around $(\pi, 0)$ and $(\pi / 2, \pi / 2)$ have been detected $\stackrel{19,20}{=}$ This phenomena is well explained in terms of the $\mathbf{k}$-dependent band-folding effect associated with an AF order which splits the band into upper and lower branches, $14,20,21,22$ leading to the two-band and/or two-gap model.

Recently, neutron scattering experiments in electrondoped cuprates have revealed that the spin response is commensurate in a substantial frequency range below a crossover frequency $\omega_{c}, 23,24,25,26,27,28$ which constitutes a distinct difference from the widely studied hourglass dispersion in the hole-doped cuprates ${ }^{29}$ Although, both scenarios mentioned above can account for the nonmonotonic gap behavior of the electron-doped cuprates, a comparative study on the spin dynamics between the two scenarios is deserved to demonstrate the possible differences and therefore serve to select the reasonable model for electron-doped high- $T_{c}$ cuprates.

In this paper, we investigate the spin dynamics in the coexisting phase of the $\mathrm{AF}$ and the $d_{x^{2}-y^{2} \text {-wave } \mathrm{SC} \text { or- }}$ ders. The calculation is based on a self-consistent determination of the QP dispersion, the AF order and the $\mathrm{SC}$ gap at the slave-boson mean-field level of the $t-t^{\prime}-t^{\prime \prime}-J$ model. It is shown that the AF and SC orders compete and coexist in a substantial doping range in the underdoped regime. The spin response is commensurate below a crossover frequency $\omega_{c}$ for all dopings considered, and it becomes incommensurate when the frequency is higher than $\omega_{c}$. This result is qualitatively consistent with experiments $\frac{23,24,25,26,27,28}{}$ While in the framework of the pure SC state with $d_{x^{2}-y^{2}}$-wave and/or DP $+\mathrm{HH}, \underline{30,31,32}$, though an extended region of a commensurate spin fluctuation also exists, it evolves into an incommensurate spin fluctuation at low frequencies, which is not consistent experiments. Therefore, our result suggests that the inclusion of the two-band effect resulting from the coexisting $\mathrm{AF}$ and $\mathrm{SC}$ orders is important to consistently account for both the spin dynamics and the non-monotonic 
gap behavior in the electron-doped cuprates.

The paper is organized as follows. In Sec. II, we introduce the theoretical model and carry out the analytical calculations. In Sec. III, we present the numerical results with some discussions. Finally, we present the conclusion in Sec. IV.

\section{THEORETICAL MODEL}

The Hamiltonian of the two dimensional $t-t^{\prime}-t^{\prime \prime}-J$ model on a square lattice is written in the form,

$$
\begin{aligned}
H= & -t \sum_{<i j>, \sigma}\left(c_{i \sigma}^{\dagger} c_{j \sigma}+H . c .\right)-t_{1} \sum_{<i j>2, \sigma}\left(c_{i \sigma}^{\dagger} c_{j \sigma}+H . c .\right)-t_{2} \sum_{<i j>3, \sigma}\left(c_{i \sigma}^{\dagger} c_{j \sigma}+H . c .\right) \\
& +J \sum_{<i j>}\left(\mathbf{S}_{i} \cdot \mathbf{S}_{j}-\frac{1}{4} n_{i} n_{j}\right)-\mu_{0} \sum_{<i>, \sigma} c_{i \sigma}^{\dagger} c_{i \sigma} .
\end{aligned}
$$

Where the summations $<i j>,<i j>_{2},<i j>_{3}$ run over the nearest-neighbor(n-n), the next-n·n, and the third-n.n pairs respectively, $\mathbf{S}_{i}$ is the spin on site $i$. This Hamiltonian can be used to model both hole-doped and electron-doped systems after a particle-hole transformation. For electron doping, one has $t<0, t_{1}>0$ and $t_{2}<0$. The slave-boson mean-field theory (SBMFT) is used to decouple the electron operators $c_{i \sigma}$ to bosons $b_{i}$ carrying the charge and fermions $f_{i \sigma}$ representing the spin. Then, the local constraint $b_{i}^{\dagger} b_{i}+\sum_{i \sigma} f_{i \sigma}^{\dagger} f_{i \sigma}=1$ is satisfied averagely at the mean-field (MF) level. We choose the spinon pairing order $\Delta_{i j}=<f_{i \uparrow} f_{j \downarrow}-f_{i \downarrow} f_{j \uparrow}>= \pm \Delta$ , where $\Delta_{i j}=\Delta(-\Delta)$ for bond $\langle i j\rangle$ along $x(y)$ direction, the uniform bond order $\chi_{i j}=\sum_{\sigma}<f_{i \sigma}^{\dagger} f_{j \sigma}>=\chi$, the AF order $<f_{i \uparrow}^{\dagger} f_{i \uparrow}-f_{i \downarrow}^{\dagger} f_{i \downarrow}>/ 2=(-1)^{i} m$, and replace $b_{i}$ by $\left\langle b_{i}\right\rangle=\sqrt{x}$ due to boson condensation. After the Fourier transformation, the mean-field (MF) Hamiltonian can be written in the Nambu representation,

$H=\sum_{\mathbf{k}} C^{\dagger}(\mathbf{k}) \hat{A}(\mathbf{k}) C(\mathbf{k})+2 N J\left(\chi^{2}+m^{2}+\Delta^{2} / 2\right)-N \mu$,

here, the Nambu operator $C^{\dagger}(\mathbf{k}) \stackrel{(2)=}{=}$ $\left(f_{\mathbf{k} \uparrow}^{\dagger}, f_{\mathbf{k}+\mathbf{Q} \uparrow}^{\dagger}, f_{-\mathbf{k} \downarrow}, f_{-\mathbf{k}-\mathbf{Q} \downarrow}\right)$, and

$$
\hat{A}(\mathbf{k})=\left(\begin{array}{cccc}
\epsilon_{\mathbf{k}} & -2 J m & -J \Delta_{\mathbf{k}} & 0 \\
-2 J m & \epsilon_{\mathbf{k}+\mathbf{Q}} & 0 & J \Delta_{\mathbf{k}} \\
-J \Delta_{\mathbf{k}} & 0 & -\epsilon_{\mathbf{k}} & -2 J m \\
0 & J \Delta_{\mathbf{k}} & -2 J m & -\epsilon_{\mathbf{k}+\mathbf{Q}}
\end{array}\right),
$$

where, $\epsilon_{\mathbf{k}}=(-2 t x-J \chi)\left(\cos k_{x}+\cos k_{y}\right)-$ $4 t_{1} x \cos k_{x} \cos k_{y}-2 t_{2} x\left(\cos 2 k_{x}+\cos 2 k_{y}\right)-\mu$ and $\Delta_{\mathbf{k}}=$ $\Delta\left(\cos k_{x}-\cos k_{y}\right) . \mu$ is the renormalized chemical potential, $N$ is the total number of lattice sites, and $\mathbf{Q}=(\pi, \pi)$ is the $\mathrm{AF}$ momentum. Note that the wave vector $\mathbf{k}$ is restricted to the magnetic Brillouin zone (MBZ) in all follows.

Diagonalizing of the Hamiltonian (2) by an unitary matrix $\hat{U}(\mathbf{k})$ leads to four energy bands $E_{1}(\mathbf{k})=E_{\mathbf{k}}^{+}$,
$E_{2}(\mathbf{k})=E_{\mathbf{k}}^{-}, E_{3}(\mathbf{k})=-E_{\mathbf{k}}^{-}, E_{4}(\mathbf{k})=-E_{\mathbf{k}}^{+}$, with

$$
E_{\mathbf{k}}^{ \pm}=\sqrt{\left(\xi_{\mathbf{k}}^{ \pm}\right)^{2}+\left(J \Delta_{\mathbf{k}}\right)^{2}}
$$

where $\xi_{\mathbf{k}}^{ \pm}=\epsilon_{\mathbf{k}}^{+} \pm \sqrt{\left(\epsilon_{\mathbf{k}}^{-}\right)^{2}+4 J^{2} m^{2}}$ with $\epsilon_{\mathbf{k}}^{ \pm}=\left(\epsilon_{\mathbf{k}} \pm\right.$ $\left.\epsilon_{\mathbf{k}+\mathbf{Q}}\right) / 2$. And the free energy is written down (Boltzmann constant $k_{B}=1$ ),

$F=-2 T \sum_{\mathbf{k}, \nu= \pm} \ln \left[2 \cosh \left(\frac{E_{\mathbf{k}}^{\nu}}{2 T}\right)\right]-\mu N+2 N J\left(\chi^{2}+m^{2}+\Delta^{2} / 2\right)$.

The MF order parameters $\chi, \Delta, m$ and the chemical potential $\mu$ for different dopings $x$ can be calculated from the self-consistent equations obtained by $\partial F / \partial \chi=0$, $\partial F / \partial \Delta=0, \partial F / \partial m=0$, and $\partial F / \partial \mu=-N(1-x)$, respectively. The magnitudes of the parameters are chosen as $t=-3.0 J, t_{1}=1.02 J, t_{2}=-0.51 J$ and $J=100$ meV to model the Fermi surface observed in ARPES experiment $\frac{19,20}{10}$ by,

Then, the bare spin susceptibility (transverse) is given

$$
\chi_{0}^{ \pm}\left(\mathbf{q}, \mathbf{q}^{\prime}, \tau\right)=\frac{1}{N}<S_{\mathbf{q}}^{+}(\tau) S_{-\mathbf{q}^{\prime}}^{-}(0)>_{(0)},
$$

where $\langle\cdots\rangle_{(0)}$ means thermal average over the eigenstates of $H, S_{\mathbf{q}}^{+}=\sum_{k} f_{\mathbf{k}+\mathbf{q} \uparrow}^{+} f_{\mathbf{k} \downarrow}$ is the spin operator. Considering that $\mathbf{k}$ is restricted to the MBZ, an explicit calculation shows that the spin susceptibility should be expressed in the following matrix form,

$$
\hat{\chi}_{0}^{ \pm}(\mathbf{q}, \omega)=\left(\begin{array}{cc}
\chi_{0}^{ \pm}(\mathbf{q}, \mathbf{q}, \omega) & \chi_{0}^{ \pm}(\mathbf{q}, \mathbf{q}+\mathbf{Q}, \omega) \\
\chi_{0}^{ \pm}(\mathbf{q}+\mathbf{Q}, \mathbf{q}, \omega) & \chi_{0}^{ \pm}(\mathbf{q}+\mathbf{Q}, \mathbf{q}+\mathbf{Q}, \omega)
\end{array}\right)
$$

where, the nondiagonal correlation function $\chi_{0}^{ \pm}$with $\mathbf{q}^{\prime}=$ $\mathbf{q}+\mathbf{Q}$ arises due to the umklapp process. The matrix elements of the bare spin susceptibility, which come from the particle-hole $(p-h)$ excitations, are given by, 


$$
\begin{aligned}
\chi_{0}^{ \pm}(\mathbf{q}, \omega)_{\eta \eta^{\prime}}= & \frac{1}{N} \sum_{i, j=1}^{2} \sum_{m, n=1}^{2} \sum_{\mathbf{k}}\left[a_{1} \frac{f\left(E_{m}(\mathbf{k})\right)-f\left(E_{n}(\mathbf{k}+\mathbf{q})\right)}{\omega+E_{n}(\mathbf{k}+\mathbf{q})-E_{m}(\mathbf{k})+i \Gamma}+a_{2} \frac{f\left(E_{n}(\mathbf{k}+\mathbf{q})\right)-f\left(E_{m}(\mathbf{k})\right)}{\omega-E_{n}(\mathbf{k}+\mathbf{q})+E_{m}(\mathbf{k})+i \Gamma}\right. \\
& \left.+b_{1} \frac{1-f\left(E_{m}(\mathbf{k})\right)-f\left(E_{n}(\mathbf{k}+\mathbf{q})\right)}{\omega+E_{n}(\mathbf{k}+\mathbf{q})+E_{m}(\mathbf{k})+i \Gamma}+b_{2} \frac{f\left(E_{m}(\mathbf{k})\right)+f\left(E_{n}(\mathbf{k}+\mathbf{q})\right)-1}{\omega-E_{n}(\mathbf{k}+\mathbf{q})-E_{m}(\mathbf{k})+i \Gamma}\right],
\end{aligned}
$$

where, $f\left(E_{\mathbf{k}}\right)$ is the Fermi function and

$$
\begin{aligned}
& a_{1}=U_{i n}^{*}(\mathbf{k}+\mathbf{q}) U_{\left(j+\eta^{\prime}-\eta\right) n}(\mathbf{k}+\mathbf{q}) U_{i m}(\mathbf{k}) U_{j m}^{*}(\mathbf{k})+U_{i n}^{*}(\mathbf{k}+\mathbf{q}) U_{(j+2) n}(\mathbf{k}+\mathbf{q}) U_{i m}(\mathbf{k}) U_{\left(j+2+\eta^{\prime}-\eta\right) m}^{*}(\mathbf{k}) \\
& a_{2}=U_{(i+2) n}(\mathbf{k}+\mathbf{q}) U_{\left(j+2+\eta^{\prime}-\eta\right) n}^{*}(\mathbf{k}+\mathbf{q}) U_{(i+2) m}^{*}(k) U_{(j+2) m}(\mathbf{k})+U_{(i+2) n}(\mathbf{k}+\mathbf{q}) U_{j n}^{*}(\mathbf{k}+\mathbf{q}) U_{(i+2) m}^{*}(\mathbf{k}) U_{\left(j+\eta^{\prime}-\eta\right) m}(\mathbf{k}) \\
& b_{1}=U_{i n}^{*}(\mathbf{k}+\mathbf{q}) U_{\left(j+\eta^{\prime}-\eta\right) n}(\mathbf{k}+\mathbf{q}) U_{(i+2) m}^{*}(\mathbf{k}) U_{(j+2) m}(\mathbf{k})-U_{i n}^{*}(\mathbf{k}+\mathbf{q}) U_{(j+2) n}(\mathbf{k}+\mathbf{q}) U_{(i+2) m}^{*}(\mathbf{k}) U_{\left(j+\eta^{\prime}-\eta\right) m}(\mathbf{k}) \\
& b_{2}=U_{(i+2) n}(\mathbf{k}+\mathbf{q}) U_{\left(j+2+\eta^{\prime}-\eta\right) n}^{*}(\mathbf{k}+\mathbf{q}) U_{i m}(\mathbf{k}) U_{j m}^{*}(\mathbf{k})-U_{(i+2) n}(\mathbf{k}+\mathbf{q}) U_{j n}^{*}(\mathbf{k}+\mathbf{q}) U_{i m}(\mathbf{k}) U_{\left(j+2+\eta^{\prime}-\eta\right) m}^{*}(\mathbf{k})
\end{aligned}
$$

The renormalized spin susceptibility due to the spin fluctuations is obtained via the random-phase approximation (RPA),

$$
\hat{\chi}^{ \pm}(\mathbf{q}, \omega)=\frac{\hat{\chi}_{0}^{ \pm}(\mathbf{q}, \omega)}{\hat{1}+\alpha \hat{J}_{q} \hat{\chi}_{0}^{ \pm}(\mathbf{q}, \omega)},
$$

where,

$$
\hat{J}_{q}=\left(\begin{array}{cc}
J(\mathbf{q}) & 0 \\
0 & J(\mathbf{q}+\mathbf{Q})
\end{array}\right)
$$

with $J(\mathbf{q})=J\left(\cos q_{x}+\cos q_{y}\right)$. In the coexisting phase of the AF order and SC order, $\alpha$ is taken as 1. As for the pure $\mathrm{SC}$ state with $\mathrm{DP}+\mathrm{HH}$, we choose a slightly small $\alpha=0.72$, the criteria for choosing $\alpha$ is to set the AF instability at $x=0.12$. The parameter $\Gamma=0.04 J$ is introduced to account for the QP damping rate which comes from the scattering off other fluctuations that are not included here.

\section{NUMERICAL RESULTS AND DISCUSSION}

In Fig. 1, we show the MF parameters $\chi, m$ and $\Delta$ as a function of doping $x$. For a comparison, we also show the doping dependence of the MF SC gap $\Delta_{1}$ obtained without considering the AF order by setting $m=0$. It is seen that the staggered magnetization $m$ decreases with increasing doping $x$, and goes sharply to zero at $x \approx 0.16$, which implies a phase transition from the antiferromagnetism (AFM) phase to the paramagnetic phase. The SC order parameter, on the other hand, increases its value initially up to an optimal doping level, and then decreases upon further doping, forming a generic SC dome. ${ }^{33}$ However, the SC order parameter $\Delta_{1}$ without the inclusion of the AF order exhibits a monotonic decrease with doping, which deviates obviously from the experimental observations. Furthermore, the SC order parameter $\Delta$ with an $\mathrm{AF}$ order shows a noticeable suppression compared to $\Delta_{1}$, exhibits a competitive character with the AF order. But, they also coexist in a substantial doping range. The MF phase diagram also shows that the optimal doping is rather low compared to that deduced from the experiments. This may be due to the fact the SBMFT includes only the MF value of the order parameters and treats the no-double occupancy on the average. However, the similarity of the phase diagram obtained by the SBMFT to that of the variational quantum-cluster theory 16,22 validates the SBMFT as a low energy effective theory. Here our aim is to use the MF theory as an effective model to study the effect of the AF order on the spin dynamics, and then to compare the two-band and/or two-gap model with the simple one-band model. Therefore, the relatively simple SBMFT is qualitatively enough for our purpose. We note that a similar phase diagram has been obtained before $\underline{\underline{15}}$

The doping dependence of the renormalized spin susceptibility $\operatorname{Im} \chi(\mathbf{q}, \omega)$ at a low frequency $\omega=0.04 \mathrm{~J}$ in the coexisting phase is presented in Fig. 2. In this figure, it is found that the low-energy excitations exhibit commensurate peaks for all $x$, which consists with the experiments well ${ }^{27}$. The inset shows the spin susceptibility $\operatorname{Im} \chi(\mathbf{q}, \omega)$ at doping $x=0.15$ in the pure SC state with $\mathrm{DP}+\mathrm{HH}$ which is used to reproduce a non-monotonic SC gap behavior. One can see that the spin response is incommensurate at low frequency without considering the AF order.

Detailed frequency dependence of the spin response in the coexisting phase and the pure $\mathrm{SC}$ phase with $\mathrm{DP}+\mathrm{HH}$ at doping $x=0.15$ are shown in Figs. 3(a) 
and 3(b), and Figs. 3(c) and 3(d), respectively. The difference in the low frequency regime of the two phases is more evident here. The spin fluctuation is commensurate in a substantial frequency range below a crossover frequency $\omega_{c} \approx 0.52 J$ and down to the lowest frequency considered in the coexisting phase, and it switches to be incommensurate when the frequency is higher than $\omega_{c} \approx 0.52 J$ [Figs. 3(a) and 3(b)]. This feature agrees with the neutron-scattering measurements on electrondoped cuprates that have been reported recently. ${ }^{23}$ While for the pure $\mathrm{SC}$ phase with $\mathrm{DP}+\mathrm{HH}$, the spin response is incommensurate at low frequency, then it switches to be commensurate within the intermediate frequency range, and becomes incommensurate again at higher frequency. 31 These results can be summarized in the intensity plot of the imaginary part of the renormalized spin susceptibility $\operatorname{Im} \chi(\mathbf{q}, \omega)$ as a function of frequency and momentum along $\left(\pi, q_{y}\right)$ direction, as shown in Fig. 4. In the figure, the solid line indicating the peak position is the dispersion of spin excitations. The commensurate spin fluctuation prevails below $\omega_{c}$ for the coexisting system [Fig. 4(a)]. For the pure SC phase with $\mathrm{DP}+\mathrm{HH}$, the dispersion shows a hourglass-like behavior [Fig. 4(c)], which is similar to the hole-doped one, and does not consistent with the experiments on electrondoped cuprates. 23

In the presence of the AF order, the energy band of quasiparticles is split into two bands. Therefore, the particle-hole excitations that contributed to the spin susceptibility are composed of two kinds of excitations, the intra-band and the inter-band excitations. In Fig. 5 , we present the results for the bare spin susceptibility $\chi_{0}(\mathbf{q}, \omega)$ (without the RPA correction) coming from the intra-band and the inter-band contributions, respectively. Figs. 5(a1) and 5(a2) denote the imaginary part of $\chi_{0}(\mathbf{q}, \omega)$, Figs. $5(\mathrm{~b} 1)$ and $5(\mathrm{~b} 2)$ the real part. One obvious feature is that, the intra-band contribution is zero at the AF momentum $\mathbf{Q}=(\pi, \pi)$, leading to the incommensurate spin response. It results from the fact that the coherence factor in the spin susceptibility due to the intra-band excitations, $1-\left[(2 \mathrm{Jm})^{2}-\right.$ $\left.\varepsilon_{\mathbf{k}+\mathbf{q}} \varepsilon_{\mathbf{k}}\right] /\left[\sqrt{\varepsilon_{\mathbf{k}+\mathbf{q}}^{2}+(2 J m)^{2}} \sqrt{\varepsilon_{\mathbf{k}}^{2}+(2 J m)^{2}}\right]$ [where, $\varepsilon_{\mathbf{k}}=$ $\left.(-2 t x-J \chi)\left(\cos k_{x}+\cos k_{y}\right)\right]$ is zero at $\mathbf{Q}$. While, the inter-band contribution is commensurate for all frequencies. At low frequencies, the inter-band excitations have a larger contribution to the spin susceptibility than the intra-band excitations, so the spin fluctuation is commensurate. However, with the increase of frequency, the intensity of $\operatorname{Im} \chi_{0}(\mathbf{q}, \omega)$ due to the intra-band contributions increases more rapidly than the inter-band contribution. As a result, the spin fluctuation switches from a commensurate to an incommensurate structure.

\section{CONCLUSION}

In this paper, we have investigated the spin dynamics in the electron-doped cuprates in the coexisting phase of the $d_{x^{2}-y^{2}}$-wave SC and AF orders, and compared the results with that in the dominant $d_{x^{2}-y^{2}}$-wave phase with a higher harmonics term. In the coexisting phase, we found that the spin response is commensurate in a substantial frequency range below a crossover frequency $\omega_{c}$ for all dopings considered, and it switches to be incommensurate when the frequency is higher than $\omega_{c}$. The theoretical calculations are shown to be in good agreement with the experimental measurements. However, in the dominant $d_{x^{2}-y^{2}}$-wave phase with a higher harmonics term, the dispersion is just like that of the hole-doped one, namely exhibits a hourglass-like dispersion, which is not consistent with experiments. Thus, our result suggests that the inclusion of the two-band effect is important to consistently account for both the dispersion of the spin response and the non-monotonic gap behavior in the electron-doped cuprates.

\section{Acknowledgments}

This work was supported by the National Natural Science Foundation of China (10525415), the Ministry of Science and Technology of China (973 project Grants Nos.2006CB601002,2006CB921800), and the China Postdoctoral Science Foundation (Grant No. 20080441039).

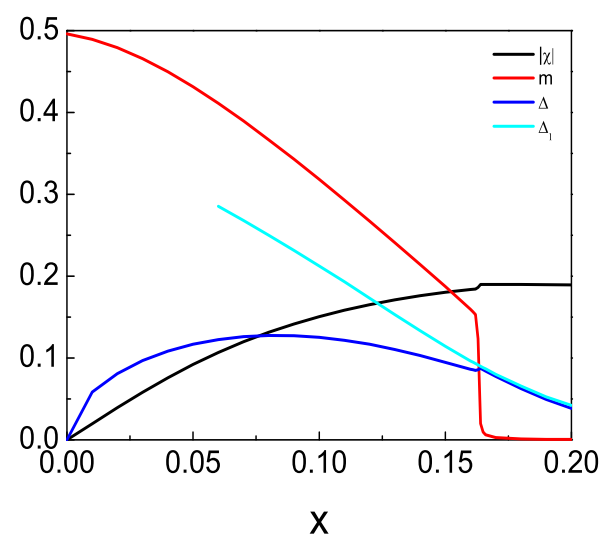

FIG. 1: (Color online) Mean-field phase diagram for $t-t^{\prime}-$ $t^{\prime \prime}-J$ model, where $\Delta_{1}$ is the superconducting order parameter without considering the AF order. The model parameters are taken as: $t=-3.0 \mathrm{~J}, t^{\prime}=1.02 \mathrm{~J}, t^{\prime \prime}=-0.51 \mathrm{~J}$.
1 C. C. Tsuei and J. R. Kirtley, Phys. Rev. Lett. 85, 182 (2000); A. D. Darminto, H. J. H. Smilde, V. Leca, D. H.
A. Blank, H. Rogalla, and H. Hilgenkamp, Phys. Rev. Lett. 94, 167001 (2005); A. Biswas, P. Fournier, M. M. Qazil- 


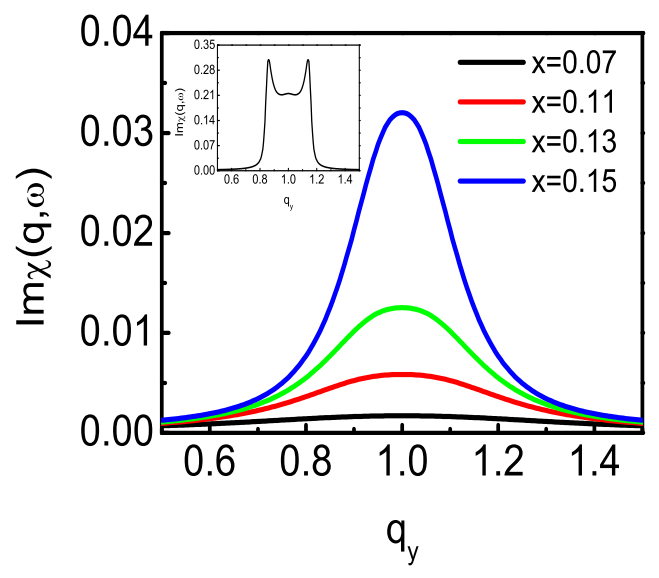

FIG. 2: (Color online) Doping dependence of $\operatorname{Im} \chi(\mathbf{q}, \omega)$ in the coexisting phase of the $\mathrm{AF}$ and $\mathrm{SC}$ order at low frequency $\omega=0.04 J$. The momentum is scanned along $\left(\pi, q_{y}\right)$. The inset shows $\operatorname{Im} \chi(q, \omega)$ at $\omega=0.04 J$ for the pure SC state with $\mathrm{DP}+\mathrm{HH}$ at doping $x=0.15$.

bash, V. N. Smolyaninova, H. Balci, and R. L. Greene, Phys. Rev. Lett. 88, 207004 (2002).

${ }^{2}$ L. Shan, Y. Huang, Y. L. Wang, S. Li, J. Zhao, P. Dai, Y. Z. Zhang, C. Ren, and H. H. Wen, Phys. Rev. B 77, 014526 (2008); A. Zimmers, Y. Noat, T. Cren, W. Sacks, D. Roditchev, B. Liang, and R. L. Greene, Phys. Rev. B 76, 132505 (2007); G.-Q. Zheng, T. Sato, Y. Kitaoka, M. Fujita, and K. Yamada, Phys. Rev. Lett. 90, 197005 (2003); J. A. Skinta, M.-S. Kim, T. R. Lemberger, T. Greibe and M. Naito, Phys. Rev. Lett. 88, 207005 (2002).

3 T. Sato, T. Kamiyama, T. Takahashi, K. Kurahashi, and K. Yamada, Science 291, 1517 (2001).

${ }^{4}$ N. P. Armitage, D. H. Lu, D. L. Feng, C. Kim, A. Damascelli, K. M. Shen, F. Ronning, Z. X. Shen, Y. Onose, Y. Taguchi, and Y. Tokura, Phys. Rev. Lett. 86, 1126 (2001).

${ }^{5}$ H. Matsui, K. Terashima, T. Sato, T. Takahashi, M. Fujita, and K. Yamada, Phys. Rev. Lett. 95, 017003 (2005).

${ }^{6}$ S. R. Park, Y. S. Roh, Y. K. Yoon, C. S. Leem, J. H. Kim, B. J. Kim, H. Koh, H. Eisaki, N. P. Armitage, and C. Kim, Phys. Rev. B 75, 060501(R) (2007).

7 G. Blumberg, A. Koitzsch, A. Gozar, B. S. Dennis, C. A. Kendziora, P. Fournier, and R. L. Greene, Phys. Rev. Lett. 88, 107002 (2002).

8 Y. Dagan, R. Beck, and R. L. Greene, Phys. Rev. Lett. 99, 147004 (2007).

9 P. Krotkov and A. V. Chubukov, Phys. Rev. Lett. 96, 107002 (2006).

10 H. Yoshimura and D. S. Hirashima, J. Phys. Soc. Jpn. 73, 2057 (2004); 74, 712 (2005).

11 V. A. Khodel, V. M. Yakovenko, M. V. Zverev, and H. Kang, Phys. Rev. B 69, 144501 (2004).

12 T. Watanabe, T. Miyata, H. Yokoyama, Y. Tanaka, and J.-I. Inoue, J. Phys. Soc. Jpn. 74, 1942 (2005).
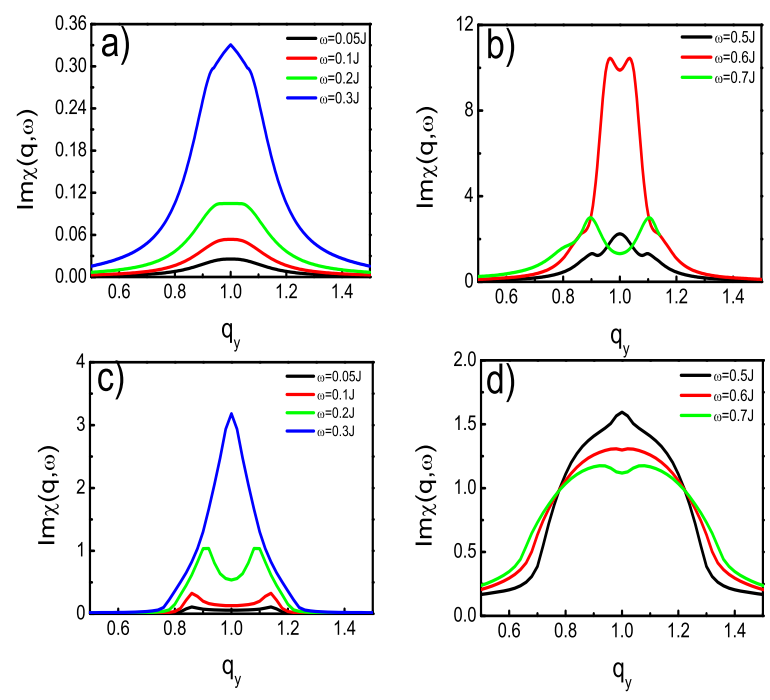

FIG. 3: (Color online) Frequency dependence of $\operatorname{Im} \chi(\mathbf{q}, \omega)$ at doping $x=0.15$. The momentum is scanned along $\left(\pi, q_{y}\right)$. (a) and (b) are in the coexistence of $\mathrm{AF}$ and SC state. (c) and (d) are in the pure $\mathrm{SC}$ state with $\mathrm{DP}+\mathrm{HH}$.

13 P. Krotkov and A. V. Chubukov, Phys. Rev. B 74, 014509 (2006).

14 Q. S. Yuan, F. Yuan, and C. S. Ting, Phys. Rev. B 73, 054501 (2006); Q. S. Yuan, X. Z. Yan, and C. S. Ting, ibid. 74, 214503 (2006).

15 W. Yuan, H.-D. Lü, H.-Y. Lu, and Q.-H. Wang, Phys. Rev. B 77, 064515 (2008).

16 T. Das, R. S. Markiewicz, and A. Bansil, Phys. Rev. B 74, 020506(R) (2006); M. Aichhorn, E. Arrigoni, M. Potthoff, and W. Hanke, ibid. 74, 024508 (2006).

17 H. G. Luo and T. Xiang, Phys. Rev. Lett. 94, 027001 (2005).

18 C. S. Liu, H. G. Luo, W. C. Wu, and T. Xiang, Phys. Rev. B 73, 174517 (2006).

19 N. P. Armitage, F. Ronning, D. H. Lu, C. Kim, A. Damascelli, K. M. Shen, D. L. Feng, H. Eisaki, Z.-X. Shen, P. K. Mang, N. Kaneko, M. Greven, Y. Onose, Y. Taguchi, and Y. Tokura, Phys. Rev. Lett. 88, 257001 (2002).

${ }^{20}$ H. Matsui, K. Terashima, T. Sato, T. Takahashi, S.-C. Wang, H.-B. Yang, H. Ding, T. Uefuji, and K. Yamada, Phys. Rev. Lett. 94, 047005 (2005).

21 C. Kusko, R. S. Markiewicz, M. Lindroos, and A. Bansil, Phys. Rev. B 66, 140513(R) (2002).

22 D. Sénéchal, P.-L. Lavertu, M.-A. Marois, and A.-M. S. Tremblay, Phys. Rev. Lett. 94, 156404 (2005).

23 S. D. Wilson, S. Li, H. Woo, P. Dai, H. A. Mook, C. D. Frost, S. Komiya, and Y. Ando, Phys. Rev. Lett. 96, 157001 (2006)

${ }^{24}$ K. Yamada, K. Kurahashi, T. Uefuji, S. Park, S.-H. Lee, and Y. Endoh, Phys. Rev. Lett. 90, 137004 (2003) 

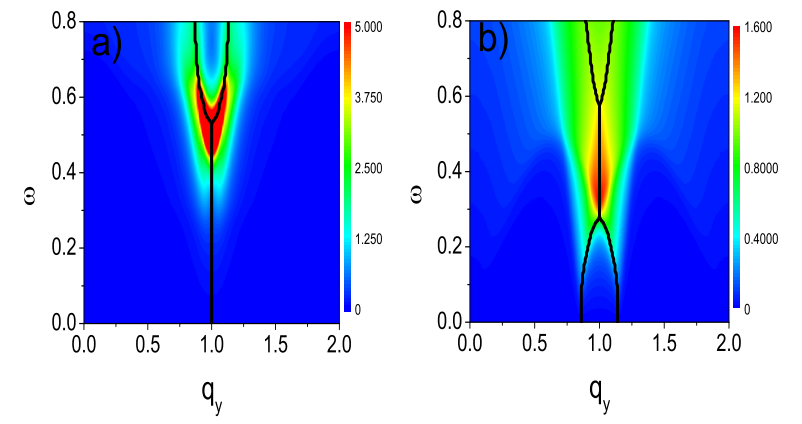

FIG. 4: (Color online) Intensity plot of $\operatorname{Im} \chi(\mathbf{q}, \omega)$ as a function of frequency $\omega$ and momentum $\mathbf{q}$ at doping $x=0.15$. The momentum is scanned along $\left(\pi, q_{y}\right)$. The solid line is the peak position. (a) is in the coexistence of AF and SC state and (b) in the pure $\mathrm{SC}$ state with $\mathrm{DP}+\mathrm{HH}$.
${ }^{25}$ H. J. Kang, P. Dai, H. A. Mook, D. N. Argyriou, V. Sikolenko, J. W. Lynn, Y. Kurita, S. Komiya, and Y. Ando, Phys. Rev. B 71, 214512 (2005)

26 S. D. Wilson, S. Li, P. Dai, W. Bao, J. Chung, H. J. Kang, S. H. Lee, S. Komiya, Y. Ando, and Q. Si, Phys. Rev. B 74, 144514 (2006)

27 M. Fujita, M. Matsuda, S.-H. Lee, M. Nakagawa, and K. Yamada, Phys. Rev. Lett. 101, 107003 (2008)

28 E. M. Motoyama, G. Yu, I. M. Vishik, O. P. Vajk, P. K. Mang, and M. Greven, Nature 445, 186 (2007).

29 P. Bourges, Y. Sidis, H. F. Fong, L. P. Renault, J. Bossy, A. Ivanov, and B. Keimer, Science 288, 1234 (2000); P. Dai, H. A. Mook, R. D. Hunt, and F. Dogan, Phys. Rev. B 63, 054525 (2001); S. M. Hayden, H. A. Mook, P. Dai, T. G. Perring, and F. Dogan, Nature 429, 531 (2004); J. M. Tranquada, H. Woo, T. G. Perring, H. Goka, G. D. Gu, G. Xu, M. Fujita, and K. Yamada, Nature 429, 534 (2004).

30 J. X. Li, J. Zhang, and J. Luo, Phys. Rev. B 68, 224503 (2003).

31 C. P. Chen, T. Zhou and J. X. Li, Physica C 469, 234 (2009).

32 K. Krüger, S. D. Wilson, L. Shan, S. Li, Y. Huang, H.-H. Wen, S.-C Zhang, P. Dai, J. Zaanen, Phys. Rev. B 76, 094506 (2007).

33 P. A. Lee, N. Nagaosa, and X.-G. Wen, Rev. Mod. Phys. 78, 000017 (2006). 

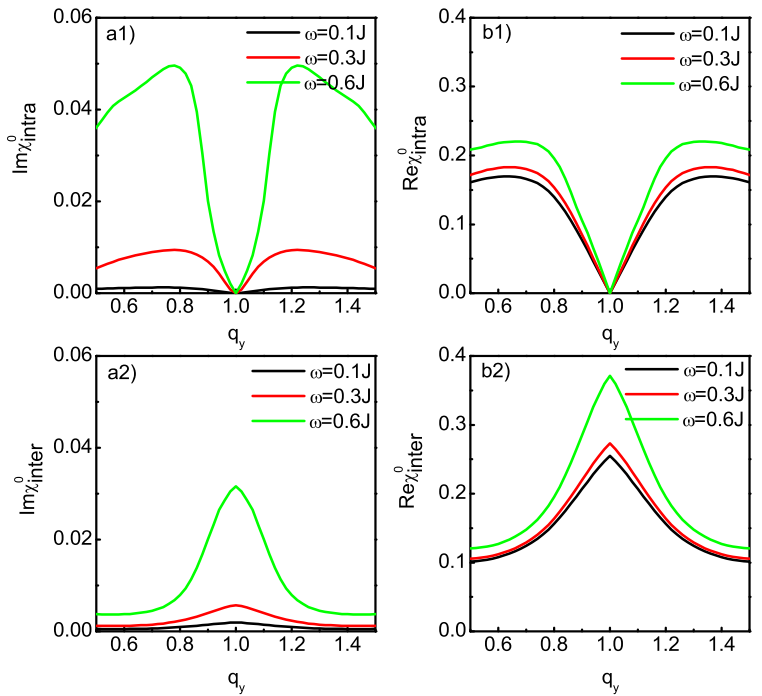

FIG. 5: (Color online) Frequency dependence of the intraand inter-band contributions to the bare spin susceptibility $\chi_{0}(\mathbf{q}, \omega)[(\mathrm{a} 1)$ and $(\mathrm{a} 2)$ denote the imaginary part, (b1) and (b2) the real part] in the coexistence of $\mathrm{AF}$ and $\mathrm{SC}$ state at doping $x=0.15$. (a1) and (b1) show the intra-band contributions, and (a2) and (b2) the inter-band contribution. 